\title{
Many-Body Effects in Hyperfine Interactions in ${ }^{205} \mathrm{~Pb}^{+}$
}

\author{
Sonjoy Majumder ${ }^{1},{ }^{\ddagger}$ B. K. Sahoo ${ }^{2}$, R. K. Chaudhuri ${ }^{3}$, B. P. Das $^{3}$ and D. Mukherjee ${ }^{4}$ \\ ${ }^{1}$ Department of Physics, Indian Institute of Technology-Madras, Chennai-600 036, India \\ ${ }^{2}$ Max Planck Insttitute for the Physics of Complex Systems, D-01187 Dresden, Germany \\ ${ }^{3}$ Non-Accelerator Particle Physics Group, Indian Institute of Astrophysics, Bangalore-34, India \\ ${ }^{4}$ Department of Physical Chemistry, \\ Indian Association for Cultivation of Science, Calcutta-700 032, India
}

(Dated: November 21, 2018)

\begin{abstract}
Ab initio calculations have been carried out to study the magnetic dipole and electric quadrupole hyperfine structure constants of ${ }^{205} \mathrm{~Pb}^{+}$. Many-body effects have been considered to all orders using the relativistic coupled-cluster theory in the singles, doubles and partial triples approximation. The trends of these effects are found to be different from atomic systems that have been studied earlier.
\end{abstract}

\section{INTRODUCTION}

With the advent of ion trapping techniques, it has become possible to perform high precision measurements of different atomic properties; including hyperfine structure constants for the ground and low-lying excited states of stable isotopes 1, 2]. Studies of these interactions have served as stringent tests of relativistic many-body theories. It has been found that the behavior of electron correlation in the hyperfine interactions in the $d$-states is substantially different from those of $s$ and $p$ - states 3 . The underlying reason for this is the strong and unusual core-polarization effects associated with the former.

The work presented in this paper is carried out using the relativistic coupled-cluster (RCC) theory. This theory has certain features which make it suitable for the calculations of excited state properties. Prominent among them being its abilities to treat relativistic and correlation effects in the initial and final states in a rigorous and balanced way [4]. It is equivalent to all orders relativistic many-body perturbation theory. Core-polarization and pair-correlation effects which are important for such calculations are computed to all orders in this theory. It has been

\footnotetext{
‡ Electronic address: bijaya@mpipks-dresden.mpg.de
} 
successfully applied to heavy atomic systems with a single valence electron [4, 5].

${ }^{205} \mathrm{~Pb}$ is one of the stable isotopes of lead which has lifetime about $1.5 \times 10^{7}$ years. The nuclear structure of this isotope is different from the other stable isotope, ${ }^{207} \mathrm{~Pb}$. It's nuclear magnetic moment and electric quadrupole moment are non-zero. Theoretical studies of the magnetic dipole and electric quadrupole hyperfine structure constants of ${ }^{205} \mathrm{~Pb}^{+}$are the focus of the present work.

There are relatively few measurements of the hyperfine structure constants for the excited states of heavy atoms and ions. It is certainly worthwhile to perform highly correlated calculations of these quantities for $\mathrm{Pb}^{+}$as it could be a promising candidate for the observation of parity nonconservation [6]. This theoretical effort could motivate experimentalists to carry out high precision measurements of ${ }^{205} \mathrm{~Pb}^{+}$.

In section II of this paper, we give an outline of the RCC theory and in section III we discuss the pertinent details of the calculation. The results of our calculations are presented and discussed in section III and finally we make some concluding remarks in section IV.

\section{THEORY}

In order to obtain the RCC wavefunction for $\mathrm{Pb}^{+}$, we require the closed-shell $\mathrm{Pb}^{++}$wavefunction, which is given in terms of the lowest order Dirac-Fock (DF) wavefunction $\left|\Phi_{D F}\right\rangle$, as

$$
|\Psi\rangle=\Omega\left|\Phi_{D F}\right\rangle,
$$

where in conventional many-body perturbation theory, the wave operator, $\Omega$ is expressed in powers

of the residual interaction, $V_{e s}=\sum_{i<j} \frac{1}{r_{i j}}-\sum_{i} U_{D F}\left(r_{i}\right)$. This results in a hierarchy of approximations for the correlation energy and the wavefunction.

The coupled cluster theory is based on the following exponential ansatz for the closed-shell wave operator [7]

$$
\Omega=N[\exp (T)]
$$

where the cluster operator $T$ is expressed in terms of the connected diagrams of the wave operator. The operator $T$ also can be expressed in terms of the cluster operators $T_{n}$ corresponding to different orders of excitations $n$ of the core electrons from the DF state, $\left|\Phi_{D F}\right\rangle$, explicitly defined by,

$$
\begin{aligned}
T & =T_{1}+T_{2}+\ldots \\
& =\sum_{a p} a_{p}^{\dagger} a_{a} t_{a}^{p}+\frac{1}{4} \sum_{a b p q} a_{p}^{+} a_{q}^{+} a_{b} a_{a} t_{a b}^{p q}+\ldots
\end{aligned}
$$


with $a, b, c, . .(p, q, r, .$.$) representing occupied (unoccupied) orbitals. t_{a}^{p}$ is the cluster amplitude corresponding to the single excitation from the orbital $a$ to $p$ and so on. Termination of the series at $T_{2}$, results in the coupled cluster theory with single and double excitations (CCSD). The contribution from the Breit interaction [8] which is four orders of magnitude smaller than the Coulomb interaction has been neglected in the present work.

For a single valence atomic system the wavefunction in the RCC method can be written as [7, 9$]$

$$
\left|\Psi_{v}\right\rangle=e^{T}\left\{1+S_{v}\right\}\left|\Phi_{v}\right\rangle
$$

with the new reference state

$$
\left|\Phi_{v}\right\rangle=a_{v}^{\dagger}\left|\Phi_{D F}\right\rangle
$$

for the given valence electron $v ; S_{v}$ represents excitation operators which excite at least the valence electron. The explicit form of this operator can be written as

$$
\begin{aligned}
S_{v} & =S_{1 v}+S_{2 v}+\ldots \\
& =\sum_{p \neq v} a_{p}^{+} a_{v} s_{v}^{p}+\frac{1}{2} \sum_{b p q} a_{p}^{+} a_{q}^{+} a_{b} a_{v} s_{v b}^{p q}+\ldots
\end{aligned}
$$

An approximate treatment of the triple excitations to the CCSD method is included by contracting the residual Coulomb operator, which effectively forms a two-body operator, and the double-excitation operators $T_{2}$ and $S_{2 v}$ 10], thereby defining the $\operatorname{CCSD}(\mathrm{T})$ approximation

$$
S_{v b c}^{p q r}=\frac{{\widehat{V_{e s} T}}_{2}+\widehat{V}_{e s} \widehat{S}_{2 v}}{\epsilon_{v}+\epsilon_{b}+\epsilon_{c}-\epsilon_{p}-\epsilon_{q}-\epsilon_{r}},
$$

where $\epsilon_{i}$ is the orbital energy of the corresponding $i$ 'th electron.

\section{METHOD OF CALCULATION}

For computational simplicity, the $T$ amplitudes are solved first for the closed-shell $\mathrm{Pb}^{++}$and then the valence electron is attached to calculate the open-shell wavefunctions. The matrix equations for solving the correlation energy and the $T$ amplitudes are given by

$$
\begin{array}{r}
\left\langle\Phi_{D F}\left|\overline{\mathrm{H}_{N}}\right| \Phi_{D F}\right\rangle=\Delta E_{\text {corr }} \\
\left\langle\Phi_{D F}^{*}\left|\overline{\mathrm{H}_{N}}\right| \Phi_{D F}\right\rangle=0,
\end{array}
$$

where $\mathrm{H}_{N}$ is the normal ordering Hamiltonian which can be written as $\mathrm{H}_{N}=\mathrm{f}_{N}+\mathrm{V}_{N}$, sum of one-body and two-body terms. In the above equation, $\overline{\mathrm{H}_{N}}$ is defined as $\overline{\mathrm{H}_{N}}=e^{-T} \mathrm{H}_{N} e^{T}, \Delta E_{\text {corr }}$ is the correlation energy and $\left|\Phi_{D F}^{*}\right\rangle$ corresponds to excited states from $\left|\Phi_{D F}\right\rangle$. 
For the open-shell RCC calculations, the $S_{v}$ operators are solved using the following equations

$$
\begin{array}{r}
\left\langle\Phi_{v}\left|\overline{\mathrm{H}_{N}}\left\{1+S_{v}\right\}\right| \Phi_{v}\right\rangle=-\Delta E_{v} \\
\left\langle\Phi_{v}^{*}\left|\overline{\mathrm{H}_{N}}\left\{1+S_{v}\right\}\right| \Phi_{v}\right\rangle=-\Delta E_{v}\left\langle\Phi_{v}^{*}\left|\left\{S_{v}\right\}\right| \Phi_{v}\right\rangle,
\end{array}
$$

where $\Delta E_{v}$ is the ionization potential (IP) energy of the corresponding valence electron $v$. Similarly $\left|\Phi_{v}^{*}\right\rangle$ are the excited states from $\left|\Phi_{v}\right\rangle$.

The relativistic hyperfine Hamiltonian is given by [1]

$$
H_{h f s}=\sum_{k} \mathbf{M}^{(\mathbf{k})} \cdot \mathbf{T}^{(\mathbf{k})}
$$

where $\mathbf{M}^{(\mathbf{k})}$ and $\mathbf{T}^{(\mathbf{k})}$ are spherical tensor operators of rank k. In first-order perturbation theory, hyperfine energies $E_{h f s}(J)$ of the fine-structure state $\left|J M_{J}\right\rangle$ are expectation values of the hyperfine interaction Hamiltonian. Details of the expression are given by Chang and Childs [11]. The magnetic dipole and electric quadrupole hyperfine energies are defined by

$$
E_{M 1}=A K / 2
$$

and

$$
E_{Q 2}=\frac{B}{2} \frac{3 K(K+1)-4 I(I+1) J(J+1)}{2 I(2 I-1) 2 J(2 J-1)},
$$

respectively. Here $I$ and $J$ being the total angular momentum of the nucleus and the electron state, respectively, and $K=2\langle I \cdot J\rangle$. The magnetic dipole hyperfine constant $A$ and electric quadrupole hyperfine constant $B$ are defined as

$$
A=\mu_{N} g_{I} \frac{\left\langle J|| T^{(1)} \| J\right\rangle}{\sqrt{J(J+1)(J+1)}}
$$

and

$$
B=2 e Q\left[\frac{2 J(2 J-1)}{(2 J+1)(2 J+2)(2 J+3)}\right]^{1 / 2}\left\langle J\left\|T^{(2)}\right\| J\right\rangle,
$$

respectively, where $\mu_{N}$ is Bohr magneton, $g_{I}=\mu_{I} / I$ with $\mu_{I}$ and $I$ are the nuclear dipole moment and spin, and $Q$ is nuclear quadrupole moment.

The expectation value for a general one particle operator $O$ in a given valence electron $(v)$ state can be expressed in RCC theory as

$$
\begin{aligned}
\langle O\rangle_{v} & =\frac{\left\langle\Psi_{v}|O| \Psi_{v}\right\rangle}{\left\langle\Psi_{v} \mid \Psi_{v}\right\rangle} \\
& =\frac{\left\langle\Phi_{v}\left|\left\{1+S_{v}^{\dagger}\right\} e^{T^{\dagger}} O e^{T}\left\{1+S_{v}\right\}\right| \Phi_{v}\right\rangle}{1+N_{v}} \\
& =\frac{\left\langle\Phi_{v}\left|\left\{1+S_{v}^{\dagger}\right\} \bar{O}\left\{1+S_{v}\right\}\right| \Phi_{v}\right\rangle}{1+N_{v}},
\end{aligned}
$$


where we define

$$
\bar{O}=e^{T^{\dagger}} O e^{T}
$$

and

$$
\begin{aligned}
N_{v} & =\left\langle\Phi_{v}\left|S_{v}^{\dagger}\left[e^{T^{\dagger}} e^{T}\right]+S_{v}^{\dagger}\left[e^{T^{\dagger}} e^{T}\right] S_{v}+\left[e^{T^{\dagger}} e^{T}\right] S_{v}\right| \Phi_{v}\right\rangle \\
& =\left\langle\Phi_{v}\left|S_{v}^{\dagger} \overline{n_{v}}+S_{v}^{\dagger} \overline{n_{v}} S_{v}+\overline{n_{v}} S_{v}^{\dagger}\right| \Phi_{v}\right\rangle
\end{aligned}
$$

For computational simplicity we evaluate the matrix elements of any operator in two steps. We expand $\bar{O}$ using Wick's general theorem [7] as

$$
\bar{O}=\left(e^{T^{\dagger}} O e^{T}\right)_{f . c .}+\left(e^{T^{\dagger}} O e^{T}\right)_{o . b .}+\left(e^{T^{\dagger}} O e^{T}\right)_{t . b .}+\ldots .
$$

where we have used the abbreviations f.c., o.b. and t.b. for fully contracted, effective one-body and effective two-body terms respectively. In this expansion of $\bar{O}$, the effective one-body terms are computed keeping terms of the form of

$$
\bar{O}_{o . b .}=O+T^{\dagger} O+O T+T^{\dagger} O T
$$

The calculation procedure for these terms are given by Geetha et al [5]. They are finally connected with $S_{v}$ and $S_{v}^{\dagger}$ operators in the evaluation of properties . Contributions due to the effective two-body terms from $\bar{O}$ are constructed using the procedure shown diagrammatically in our earlier works [5, 12] and computed directly during the calculation of properties. The following The following types of terms are considered for the construction of the effective two-body terms

$$
\bar{O}_{t . b .}=O T_{1}+T_{1}^{\dagger} O+O T_{2}+T_{2}^{\dagger} O
$$

Other effective terms correspond to higher orders in the residual Coulomb interaction and are neglected in the present calculation. A similar procedure has been followed to account for the normalization factor.

The contributions from the normalization factors for the corresponding valence electron $v$ that are given in in tables III and IV, were obtained using the following relations:

$$
N o r m=\left\langle\Psi_{v}|O| \Psi_{v}\right\rangle\left\{\frac{1}{1+N_{v}}-1\right\} .
$$

\section{RESULTS AND DISCUSSIONS}

The starting point of our calculations is the generation of DF orbitals for ${ }^{205} \mathrm{~Pb}^{++}$. These orbitals are constructed as linear combinations of Gaussian type orbitals (GTOs) as given by 
Chaudhuri et al. [13]. It has been found [6] that the RCC calculations based on these kind of GTOs provide accurate $A$ - values for the low-lying states of ${ }^{207} \mathrm{~Pb}^{+}$. In the present work, we have used similar basis functions to calculate both $A$ - and $B$ - values of ground and some of the important excited states of ${ }^{205} \mathrm{~Pb}^{+}$.

In table I, we present $\mathrm{A}$ and $\mathrm{B}$ hyperfine structure constants of the low-lying states of $\mathrm{Pb}^{+}$. We use the Lande nuclear g-factor, $g_{I}=0.28468$ to calculate $A$ and nuclear quadrupole moment, $Q$ $=0.234$ to calculate B [14]. From the differences of the DF and RCC results given in table I, it is evident that for both the hyperfine constants A and B the electron correlation effects vary from $(10-290) \%$.

TABLE I: $A$ and $B$ results of ${ }^{205} \mathrm{~Pb}^{+}$using $\mathrm{DF}$ and RCC methods.

\begin{tabular}{|c|c|c|c|c|c|c|c|c|}
\hline & $6 p_{1 / 2}$ & $6 p_{3 / 2}$ & $7 s_{1 / 2}$ & $7 p_{1 / 2}$ & $7 p_{3 / 2}$ & $6 d_{3 / 2}$ & $6 d_{5 / 2}$ & $8 s_{1 / 2}$ \\
\hline \multicolumn{9}{|l|}{$\underline{A}$} \\
\hline $\mathrm{DF}$ & 2765.54 & 220.58 & 1879.06 & 476.34 & 43.84 & 19.05 & 7.53 & 687.77 \\
\hline $\mathrm{RCC}$ & 3099.5 & 149.7 & 2680.3 & 543.7 & 74.8 & -10.03 & 60.97 & 929.3 \\
\hline \multicolumn{9}{|l|}{$\underline{B}$} \\
\hline $\mathrm{DF}$ & & 377.81 & & & 75.09 & 11.08 & 13.07 & \\
\hline $\mathrm{RCC}$ & & 464.6 & & & 99.3 & 50.7 & 56.9 & \\
\hline
\end{tabular}

All the core orbitals were excited in our calculations. The core-polarization effects, which are the largest contributors to the hyperfine constants of the $6 p_{3 / 2}$ state of ${ }^{207} \mathrm{~Pb}^{+}[6]$ and the $d_{5 / 2}$ states of the alkaline earth ions [15], have been accounted to all orders through the $O S_{2 v}$ term. It was also found from the hyperfine structure studies of the $s_{1 / 2}$ and $p_{1 / 2}$ states in the alkaline earth ions that pair-correlation and core-polarization effects are important. In order to appreciate the importance of these effects in $\mathrm{Pb}^{+}$, we present their contributions in table II. Comparison of these results with their corresponding DF values from Table I, brings out some distinct many-body features of the system. The most prominent among them is the size of the core-correlation and core-polarization effects for the $d$-states. As in the case of some of the alkaline earth ions, the sign of the core-polarization effect in the $d_{5 / 2}$ state is opposite that of the DF value and the net contribution is $209 \%$ of the corresponding DF value. However, unlike those alkaline earth ions 15] the final RCC result has the same sign as the DF result. This is due to the large positive 
TABLE II: The contributions of core-correlation, core-polarization and pair-correlation of $A$ and $B$ results in ${ }^{205} \mathrm{~Pb}^{+}$.

\begin{tabular}{lrrr}
\hline \hline States & Core-corr. & Core-pol. & Pair-corr. \\
\hline & & & \\
$\mathrm{A}$ & & & 6.01 \\
$7 p_{3 / 2}$ & -0.68 & 15.17 & 9.30 \\
$6 d_{3 / 2}$ & -1.02 & 11.13 & 3.45 \\
$6 d_{5 / 2}$ & -0.48 & -15.73 & 139.92 \\
$8 s$ & 70.29 & 144.23 & \\
\hline & & & 35.55 \\
$\mathrm{~B}$ & & & 10.29 \\
$6 p_{3 / 2}$ & 29.26 & 79.19 & 5.44 \\
$7 p_{3 / 2}$ & 3.34 & 15.78 & 5.96 \\
$6 d_{3 / 2}$ & -0.65 & 17.87 & \\
$6 d_{5 / 2}$ & -0.88 & 23.46 & \\
\hline \hline
\end{tabular}

contributions from the $S_{2 v}^{\dagger} \bar{O} S_{2 v}$ term, shown in table III . Therefore, it is imperative to consider such terms to obtain accurate results.

The role of electron correlation in the hyperfine interactions in ${ }^{45} S c$ and ${ }^{89} Y$ has similarities 3] with $\mathrm{Pb}^{+}$even though those systems are neutral and have different electronic configurations. Another interesting feature of the present study on ${ }^{205} \mathrm{~Pb}^{+}$is that the RCC result of the $A$ - value of $6 d_{3 / 2}$ is of opposite in sign than that of the DF result. This trend is different from the other $d_{3 / 2}$ states in alkaline earth ions $[3,16]$. The main reason for this behavior is due to another unusual contribution from $S_{2 v}^{\dagger} \bar{O} S_{2 v}$. The core correlation effect on $B$ - values though reduces as higher excited states are considered, which is expected, but contributions to the $d$ - states are not similar to the $p$ - states. This is evident from the $B$ - values given in table II.

Table III and IV present the important effective two-body terms obtained from $\bar{O}=e^{T^{\dagger}} O e^{T}$, but they contribute very little. One can therefore justifiably ignore the higher order terms given in eqn. (3.15) and save computational time. The correlation effects of the $A$ - values of other states presented in table I, behave the same way as in ${ }^{207} \mathrm{~Pb}^{+}[\underline{6}]$. 
TABLE III: Contributions of different coupled-cluster terms to the $\mathrm{Pb}^{+}$magnetic dipole hyperfine structure constant $(A) . c c$ stands for the complex conjugate part of the corresponding terms.

\begin{tabular}{lcccc}
\hline \hline Terms & $\begin{array}{r}7 \mathrm{p}_{3 / 2} \\
\text { state }\end{array}$ & $\begin{array}{c}6 \mathrm{~d}_{3 / 2} \\
\text { state }\end{array}$ & $\begin{array}{c}6 \mathrm{~d}_{5 / 2} \\
\text { state }\end{array}$ & $\begin{array}{c}8 \mathrm{~s}_{1 / 2} \\
\text { state }\end{array}$ \\
\hline & & & & \\
$O(\mathrm{DF})$ & 43.84 & 19.05 & 7.53 & 687.77 \\
\hline $\bar{O}$ & 44.52 & 20.07 & 8.01 & 617.48 \\
$\bar{O} S_{1 v}+c c$ & 6.01 & 9.30 & 3.45 & 139.92 \\
$\bar{O} S_{2 v}+c c$ & 15.17 & 11.13 & -15.73 & 144.23 \\
$S_{1 v}^{\dagger} \bar{O} S_{1 v}$ & 0.21 & 1.09 & 0.38 & 7.92 \\
$S_{1 v}^{\dagger} \bar{O} S_{2 v}+c c$ & -0.86 & 2.02 & -2.08 & 4.85 \\
$S_{2 v}^{\dagger} \bar{O} S_{2 v}+c c$ & 11.04 & -54.93 & 68.91 & 29.74 \\
\hline
\end{tabular}

Important effective two-body terms of $\bar{O}$

\begin{tabular}{lcccc}
$S_{2 v}^{\dagger} O T_{1}+c c$ & -0.03 & -0.03 & -0.01 & 1.03 \\
$S_{2 v}^{\dagger} O T_{2}+c c$ & -0.44 & 0.74 & 0.26 & -3.61 \\
$N o r m$ & -0.69 & 0.39 & -2.21 & -10.06 \\
\hline \hline
\end{tabular}

\section{CONCLUSION}

The RCC theory has been employed to study the magnetic dipole and electric quadrupole hyperfine structure constants of the ${ }^{205} \mathrm{~Pb}^{+}$. Strong electron correlations effects are found in the $d$ - states and their behavior is different from other systems studied earlier. Experiments to measure these quantities will constitute important tests of the relativistic coupled-cluster theory.

\section{ACKNOWLEDGMENT}

We are grateful to Prof. Werth for valuable discussions and suggestions for this calculation. The calculations were carried out using the Tera-flop Supercomputer at C-DAC, Bangalore, India.

[1] X. Feng, G.-Z. Li, R. Alheit and G. Werth, Phys. Rev. A, 46, 327 (1992)

[2] W. Liu et al, Phys. Rev. Lett, 82, 711 (1999)

[3] B.K. Sahoo et al., Phys. Rev. A, 68, 040501(R) (2003); B.K. Sahoo et al, J. Phys. B,38, 4379 (2005) 
TABLE IV: Contributions of different coupled-cluster terms to the $\mathrm{Pb}^{+}$electric quadrupole hyperfine structure constant $(B)$.

\begin{tabular}{|c|c|c|c|c|}
\hline Terms & $\begin{array}{l}6 \mathrm{p}_{3 / 2} \\
\text { state }\end{array}$ & $\begin{array}{l}7 \mathrm{p}_{3 / 2} \\
\text { state }\end{array}$ & $\begin{array}{l}6 \mathrm{~d}_{3 / 2} \\
\text { state }\end{array}$ & $\begin{array}{l}6 d_{5 / 2} \\
\text { state }\end{array}$ \\
\hline$O(\mathrm{DF})$ & 377.81 & 75.09 & 11.08 & 13.07 \\
\hline $\bar{O}$ & 348.55 & 71.56 & 11.73 & 13.95 \\
\hline $\bar{O} S_{1 v}+c c$ & 35.55 & 10.29 & 5.44 & 5.96 \\
\hline $\bar{O} S_{2 v}+c c$ & 79.19 & 15.78 & 17.87 & 23.46 \\
\hline$S_{1 v}^{\dagger} \bar{O} S_{1 v}$ & 0.87 & 0.36 & 0.64 & 0.65 \\
\hline$S_{1 v}^{\dagger} \bar{O} S_{2 v}+c c$ & 2.18 & 0.62 & 2.27 & 2.75 \\
\hline$S_{2 v}^{\dagger} \bar{O} S_{2 v}+c c$ & 10.11 & 2.56 & 14.26 & 11.75 \\
\hline
\end{tabular}

Important effective two-body terms of $\bar{O}$

\begin{tabular}{lcccc}
$S_{2 v}^{\dagger} O T_{1}+c c$ & -0.72 & -0.05 & -0.02 & -0.02 \\
$S_{2 v}^{\dagger} O T_{2}+c c$ & -5.19 & -0.76 & 0.43 & 0.46 \\
Norm & -4.89 & -0.93 & -2.02 & -2.07 \\
\hline \hline
\end{tabular}

[4] B. K. Sahoo, S. Majumder, R. K. Chaudhuri, B. P. Das and D. Mukherjee, J. Phys. B, 37, 3409 (2004)

[5] Geetha Gopakumar, Holger Merlitz, Rajat Chaudhuri, B.P. Das, U.S. Mahapatra, and D. Mukherjee 66, $032505(2002)$

[6] B. K. Sahoo, R. K. Chaudhuri, B.P. Das, H. Merlitz and D. Mukherjee, Phys. Rev. A 72, 032507 (2005)

[7] I. Lindgren and J. Morrison, Atomic Many-body Theory 3, ed. G. E. Lambropoulos and H. Walther (Berlin: Springer) (1985)

[8] G. Breit, Phys. Rev. 34, 553 (1929); 34, 383 (1930); 39, 616 (1932)

[9] D. Mukherjee and S. Pal, Adv. Quantum Chem. 20, 281 (1989) 032507 (2005).

[10] A. Haque and U Kaldor, Chem. Phys. Lett. 117, 374; 120, 261 (1985)

[11] K.T. Cheng and W.J. Childs, Phys. Rev. A, 31, 2775 (1985)

[12] B. K. Sahoo, PhD thesis submitted to Mangalore University, India (2005)

[13] R. K. Chaudhuri, P. K. Panda, B. P. Das, U. S. Mahapatra, and D. Mukherjee, J. Phys. B 33, 5129 (2000)

[14] M. Anselment, W. Faubel, S. Groing, A. Hanser, G. Meisel, H. Rebel and G. Schatzz, Nucl. Phys. A, 451, $471(1986)$

[15] B. K. Sahoo, C. Sur, T. Beier, B. P. Das, R. K. Choudhuri and D. Mukherjee, submitted to PRL (2006)

[16] C. Sur, B. K. Sahoo, R. K. Chaudhuri, B. P. Das and D. Mukherjee, Eur. Phys. J. D 32, 25 (2005) 